\title{
EDITORIAL
}

\section{Guidelines: yesterday, today and tomorrow}

Dear Readers,

In the past, guidelines were developed using various approaches and had various forms and various impacts on health care. In the early 1990s, a new era of clinical practice guidelines and public health guidelines was started. After a period of haphazard development, characterized by their low quality, attempts were made to standardize their development, implementation and evaluation. This new movement was led by the Institute of Medicine in the USA which in 1990 defined clinical practice guidelines as systematically developed statements to assist practitioner and patient decisions about appropriate health care for specific clinical circumstances. The definition involves the most important attributes of CPG: systematic development, appropriate health care and specific circumstances. Since then, many institutions and organizations have been established which systematically deal with guideline methodology such as the AGREE Collaboration (1998), GRADE (2000) and Guidelines International Network (2002). In today's globalized world, cooperation is much needed. The most influential international organizations in the area of evidenced-based health care (Guidelines International Network, Cochrane Collaboration, Joanna Briggs Institute, Campbell Collaboration, International Society for Evidence-Based Health Care) organize the first Global Evidence Summit in 2017.

Over that time, many problems related to guidelines have been solved, from evaluation and increased quality, to improved and standardized methodology of their development, adaptation, evaluation and implementation, participation of patients and the general public, association with health care quality indicators, to the use of alternative evidence. At the present time, guidelines commonly include conflict of interest statements, are regularly updated and mostly systematically developed as part of comprehensive national or international programs. Guideline authors use free applications such as GRADEpro GDT, RevMan 5 or the AGREE II instrument.

Currently, brand new concepts in care for the health of individuals and populations are developed. One of them is precision medicine, significantly contributed to by President Obama's speech in January 2015. This strategy is fundamentally different from some older ones such as personalized medicine. Precision medicine is concerned with prevention and individualized treatment, connecting individual genetic, environmental and experimental variables. It builds on integrated knowledge about the health status of individuals and populations with omics data using novel findings from the fields of genomics, proteomics, metabolomics, transcriptomics, epigenetics, as well as those from the study of the exposome, microbiome, social determinants of health, behavior and motivation. All information is linked to data produced by patients themselves, data contained in large health care databases, networks of research institutions and generated by continuous health care monitoring systems. The results should be better care for patients. All that is just the beginning but now is the time to prepare the inclusion of precision medicine in guidelines.

\author{
Assist. Prof. Radim Licenik, M.D. M.Sc. Ph.D. \\ Radcliffe Department of Medicine - Investigative Medicine \\ Acute Vascular Imaging Centre, John Radcliffe Hospital, University of Oxford, UK \\ Hyper Acute Stroke Unit, Northwick Park Hospital, London, UK \\ Head of the Centre for Clinical Practice Guidelines \\ Faculty of Medicine and Dentistry, Palacky University Olomouc, Czech Republic
}

